\title{
Rapid transport of muco-inert nanoparticles in cystic fibrosis sputum treated with $\mathrm{N}$-acetyl cysteine
}

\author{
Jung Soo Suk ${ }^{1}$, Samuel K Lai 2,3,4, Nicholas J Boylan², Michelle R Dawson ${ }^{5}$, Michael P \\ Boyle $^{6}$, and Justin Hanes ${ }^{\dagger, 1,2,3,7}$ \\ ${ }^{1}$ Department of Biomedical Engineering, The Johns Hopkins University School of Medicine, MD, \\ USA \\ ${ }^{2}$ Department of Chemical \& Biomolecular Engineering, The Johns Hopkins University, MD, USA \\ 3 Institute for NanoBioTechnology, The Johns Hopkins University, MD, USA \\ ${ }^{4}$ Eshelman School of Pharmaceutics, University of North Carolina at Chapel Hill, NC, USA \\ ${ }^{5}$ Georgia Institute of Technology, GA, USA \\ ${ }^{6}$ Johns Hopkins Adult Cystic Fibrosis Program, The Johns Hopkins University School of Medicine, \\ MD USA \\ ${ }^{7}$ Department of Ophthalmology, The Johns Hopkins University School of Medicine, MD, USA
}

\begin{abstract}
Aims-Sputum poses a critical diffusional barrier that strongly limits the efficacy of drug and gene carriers in the airways of individuals with cystic fibrosis (CF). Previous attempts to enhance particle penetration of CF sputum have focused on either reducing its barrier properties via mucolytics, or decreasing particle adhesion to sputum constituents by coating the particle surface with non-mucoadhesive polymers, including polyethylene glycol (PEG). Neither approach has enabled particles to penetrate expectorated sputum at rates previously observed for nonmucoadhesive nanoparticles in human cervicovaginal mucus. Here, we sought to investigate whether a common mucolytic, $N$-acetyl cysteine (NAC), in combination with dense PEG coatings on particles, can synergistically enhance particle penetration across fresh undiluted CF sputum.
\end{abstract}

Materials \& methods-We used high-resolution multiple particle tracking to measure the diffusion of uncoated and PEG-coated nanoparticles in native and NAC-treated CF sputum.

Results-We discovered that $200 \mathrm{~nm}$ particles, if densely coated with PEG, were able to penetrate CF sputum pretreated with NAC with average speeds approaching their theoretical speeds in water. Based on the rapid penetration of PEG-coated particles in NAC-treated sputum, we determined that the average spacing between sputum mesh elements was increased from $145 \pm$ $50 \mathrm{~nm}$ to $230 \pm 50 \mathrm{~nm}$ upon NAC treatment. Mathematical models based on particle transport rates suggest as much as 75 and 30\% of 200 and $500 \mathrm{~nm}$ PEG-coated particles, respectively, may penetrate a physiologically thick NAC-treated CF sputum layer within 20 min. Uncoated particles

\footnotetext{
(C) 2011 Future Medicine Ltd

${ }^{\dagger}$ Author for correspondence: The Center for Nanomedicine, The Johns Hopkins University School of Medicine, MD, USA Tel.: +1 4106146513 Fax: +1 4106146509 hanes@jhu.edu.

Ethical conduct of research

The authors state that they have obtained appropriate institutional review board approval or have followed the principles outlined in the Declaration of Helsinki for all human or animal experimental investigations. In addition, for investi gations involving human subjects, informed consent has been obtained from the participants involved.
} 
were trapped in CF sputum pretreated with NAC nearly to the same extent as in native sputum, suggesting that NAC treatment alone offered little improvement to particle penetration.

Conclusion-NAC facilitated rapid diffusion of PEG-coated, muco-inert nanoparticles in CF sputum. Our results provide a promising strategy to improve drug and gene carrier penetration in $\mathrm{CF}$ sputum, offering hope for improved therapies for CF.

\section{Keywords}

cystic fibrosis sputum; mesh spacing; multiple particle tracking; $N$-acetyl cysteine; polyethylene glycol

Mutations of the gene encoding the cystic fibrosis transmembrane conductance regulator (CFTR) protein lead to improper regulation of ions and water content of mucus in the airways of cystic fibrosis (CF) patients [1,2]. This results in the formation of markedly more viscoelastic and purulent sputum that is, at best, less efficiently cleared by ciliary action [1]. Reduced sputum clearance can lead to increasing obstruction of the airways and a massive inflammatory response triggered by bacterial infection [3]. The barrier property of CF sputum is evident by poor gene transfer with lipid- and adenovirus-based gene carriers in sputum-covered versus sputum-depleted tissues [4], which may partially account for the failures in CF gene therapy trials to date. The sputum barrier has also likely thwarted the development of localized, long-lasting antibiotic and anti-inflammatory medications, since aerosol particles capable of sustained drug release are typically trapped in the luminal CF sputum and, thus, readily removed by mucociliary and cough clearance. Diffusion chamber, fluorescence recovery after photobleaching and multiple particle tracking studies all demonstrate that the transport of conventional polymeric nanoparticles, 30-500 nm in diameter, is strongly retarded in CF sputum [5-7]. Numerous reviews on the potential of aerosol-based CF therapies cite the thick mucus layer as a challenge [8,9] and 'one of the greatest obstacles to overcome' [10]. Thus, the development of drug and gene carriers capable of efficiently penetrating the $\mathrm{CF}$ sputum barrier is sorely needed for improved $\mathrm{CF}$ therapies [11].

We have recently engineered polymeric nanoparticles as large as $200 \mathrm{~nm}$, which can penetrate CF sputum with approximately 100 -fold higher geometric mean effective diffusivity $\left(\left\langle\mathrm{D}_{\text {eff }}\right\rangle\right)$ than similarly sized conventional polystyrene (PS) particles $[12,13]$. This was accomplished by decreasing the adhesive interactions between particles and sputum constituents by densely coating particles with low-molecular-weight polyethylene glycol (PEG) [13-15]. Nevertheless, the $\left\langle\mathrm{D}_{\text {eff }}\right\rangle$ of 200 and $500 \mathrm{~nm}$ coated particles were approximately 15- and 400-fold lower in CF sputum than their rates in cervicovaginal mucus (CVM), respectively [14], most likely due to increased adhesivity and elevated steric obstruction from the sticky and dense CF sputum mesh [11,16,17].

To further enhance particle penetration across CF sputum, we sought to reduce the diffusional barrier properties of CF sputum by adjuvant mucolytics treatment. Mucolytic agents are currently used to reduce the bulk viscoelasticity of CF sputum by cleaving sputum constituents and, therefore, improve sputum clearance and lung function [18]. Commonly used mucolytic agents include recombinant human DNase (rhDNase, also known as dornase alpha or Pulmozyme ${ }^{\circledR}$ ) and $N$-acetyl cysteine (NAC; Mucomyst ${ }^{\circledR}$ ). RhDNase, which cleaves linear DNA in CF sputum, has been studied as an adjuvant for particle transport in CF sputum, but offered little to no improvement $[6,7,19]$. NAC, a mucolytic that cleaves disulfide bonds of mucin fibers, has been shown to reduce the viscosity of mucus/sputum in vitro [20] and in vivo [21]. Inhalation of a lysine salt of NAC, nacystelyn, has also been shown to be well tolerated by CF patients and to lead to a dosedependent decrease in sputum viscoelasticity and solid content [22]. Nevertheless, NAC has 
never been tested as an adjuvant to enhance particle transport in purulent CF sputum. We thus sought to test whether NAC, in combination with dense PEG coatings on particles, can further enhance particle penetration across fresh undiluted $\mathrm{CF}$ sputum.

\section{Materials \& methods CF sputum collection}

Sputum spontaneously expectorated by male and female CF patients, aged 24-55 years, was collected at the Johns Hopkins Adult Cystic Fibrosis Center, MD, USA. The procedures conformed to the ethical standards of the Johns Hopkins Medicine Institutional Review Board. Two to four samples were acquired from the weekly CF outpatient clinic, placed on ice upon collection and during transport, pooled together to minimize patient-to-patient variation, and studied the same day. The total number of individual samples used for the present study was 12 .

\section{Nanoparticle preparation \& characterization}

Fluorescent carboxyl-modified PS nanoparticles (PS-COOH), sized 200 and $500 \mathrm{~nm}$ (Molecular Probes, OR, USA), were covalently modified with $3.4 \mathrm{kDa}$ diamine $\mathrm{PEG}\left(\mathrm{NH}_{2}-\right.$ PEG-NH $\mathrm{N}_{2}$; Nektar Therapeutics, CA, USA) at a 3:1 PEG:COOH ratio, as described previously [14]. Fluorescent carboxylate-modified particles (Molecular Probes) were used as provided. Size and zeta potential (surface charge) were determined by dynamic light scattering and laser Doppler anemometry, respectively, using a ZS90 Zetasizer (Malvern Instruments, MA, USA). Size measurements were performed at $25^{\circ} \mathrm{C}$ at a scattering angle of $90^{\circ}$.

\section{Pretreatment of CF sputum with mucolytic agents}

A $1 \mathrm{M}$ solution of NAC (Sigma-Aldrich, MO, USA) was prepared and the $\mathrm{pH}$ was adjusted to approximately 7.0 with $\mathrm{NaOH}$, since its mucolytic activity is optimal with a pH of 7.0-9.0 [18]. NAC was added to CF sputum to a final concentration of $20 \mathrm{mM}$ ( $2 \%$ dilution of CF sputum), gently mixed by swirling with a pipette tip and incubated for $30 \mathrm{~min}$ at $37^{\circ} \mathrm{C}$, prior to the addition of nanoparticles.

\section{Multiple particle tracking in CF sputum}

Nanoparticles were added to approximately $350 \mu \mathrm{l}$ of CF sputum (3\% dilution; final concentration $0.008 \% \mathrm{wt} / \mathrm{vol}$ ) with or without NAC pretreatment, transferred to eight-well glass chambers (LabTek, CA, USA), and equilibrated for $2 \mathrm{~h}$ at $37^{\circ} \mathrm{C}$ prior to microscopy. The dynamics of particles were quantified using multiple particle tracking [13,14,23,24]. Briefly, $20 \mathrm{~s}$ movies at $67 \mathrm{~ms}$ temporal resolution were acquired via a silicon-intensified target camera (VE-1000, Dage-MTI, IN, USA) on an inverted epifluorescence microscope (Axiovert, Zeiss, NY, USA) with a 100X/1.4 NA objective. Movies were analyzed with Metamorph software (Universal Imaging, WI, USA) to extract $\mathrm{x}, \mathrm{y}$ positional data over time. Time-averaged mean square displacement (MSD) and $\mathrm{D}_{\text {eff }}$ for each particle were calculated as a function of time scale $(\tau)$ [23-25]. CF sputum was assumed to be locally isotropic but not necessarily homogeneous; $2 \mathrm{D}$ diffusivity is equal to $3 \mathrm{D}$ diffusivity under this condition [23]. Bulk transport properties were calculated by geometric ensemble-averaging of individual transport rates. The tracking resolution was $10 \mathrm{~nm}$, determined by tracking the displacement of particles immobilized with a strong adhesive [26]. Particle transport mechanism (immobile, hindered and diffusive) was classified as discussed previously $[24,27]$. 


\section{Determination of the mesh spacing of CF sputum}

The mesh spacing of CF sputum was estimated based on fitting an obstruction-scaling model to measured particle diffusion rates, using maximum likelihood estimation [28]. This model was previously adapted to characterize mucus mesh spacing based on virus and DNA diffusion [29,30]. The model is valid in cases where there is negligible interaction between particles and mesh, and where fluids between the mesh elements exhibit the viscous drag (microviscosity) of water. Unlike human mucus from healthy volunteers, fluids between the CF sputum mesh have markedly higher concentrations of free bio-polymers, including DNA and actin fragments [5], rendering the microviscosity significantly higher. Thus, we adapted this model by replacing the viscosity of water with the viscosity of fluids in the pores of CF sputum, which was previously estimated, based on the diffusion of dextran in CF sputum, to be approximately 2.9-fold higher than that of water [5].

\section{Scanning electron microscopy}

Scanning electron microscopy (SEM) was performed to offer visual confirmation of possible changes in sputum structure upon NAC treatment. Either native or NAC-treated CF sputum was fixed in $2 \%$ glutaraldehyde in $0.1 \mathrm{M}$ sodium cacodylate buffer $(\mathrm{pH} 7.2)$ containing 3 $\mathrm{mM} \mathrm{CaCl}_{2}$ for $1 \mathrm{~h}$, subsequently rinsed in buffer and postfixed in $1 \% \mathrm{OsO}_{4}$ in $0.1 \mathrm{M}$ sodium cacodylate buffer for $1 \mathrm{~h}$ on ice in the dark. Following a brief rinse with distilled water, samples were stained with $2 \%$ uranyl acetate for $1 \mathrm{~h}$ and then dehydrated through a graded series of ethanol solutions. Upon complete dehydration, samples were soaked in a 50:50 mixture of ethanol and hexamethyldisilazane (HMDS), followed by pure HMDS. Sputum samples were then dessicated under vacuum, sputter-coated with $20 \mathrm{~nm}$ of $\mathrm{Au} / \mathrm{Pd}$ and observed with a field-emission scanning electron microscope (LEO 1530 FE-SEM, Zeiss) operating at 1-3 kV. SEM most likely introduces artifacts that make it unsuitable for quantitative estimates of pore sizes in the sputum microstructure [13].

\section{Statistical analysis}

Statistically significant differences between two groups were analyzed with a one-tailed Student's $t$-test, assuming unequal variances.

\section{Results}

\section{Transport of PEG-coated \& uncoated $200 \mathrm{~nm}$ particles in native \& NAC-treated CF sputum}

Using PS beads as model gene/drug carriers, we first measured the penetration rates of 200 $\mathrm{nm}$ uncoated and PEG-coated PS particles (PS and PS-PEG, respectively) in native CF sputum (suPpLemENTARY Movies $1 \& 2$, see online www.futuremedicine.com/doi/suppl/10.2217/nnm.10.123). The reduced mucoadhesion of $200 \mathrm{~nm}$ PS-PEG particles compared with uncoated $200 \mathrm{~nm}$ PS particles was confirmed by the greatly improved geometric averaged ensemble MSD (<MSD $\rangle)$ over all time scales in native sputum, as well as the values that were in good agreement with our previous findings (Figure IA \& TAble 1) [13].

We hypothesized that the transport of $200 \mathrm{~nm}$ PS-PEG particles could be further improved by reducing steric hindrance imposed by the dense mesh of CF sputum. Therefore, we altered the structural/barrier properties of CF sputum by pretreating it with NAC. We found that NAC treatment further increased the particle penetration rates, as measured by $\langle\mathrm{MSD}\rangle$, of $200 \mathrm{~nm}$ PEG-coated particles by approximately tenfold over the same particles in native sputum (at $\tau=1 \mathrm{~s}$; FGURE IA \& SUPPLEMENTARY MOVIE 3$)(\mathrm{p}<0.05)$. Importantly, the average transport rate of $200 \mathrm{~nm}$ PS-PEG particles in NAC-treated sputum corresponds to a speed only approximately eightfold slower than their theoretical speeds in water $(\tau=1 \mathrm{~s})$ (TABLE 1$)$. We also evaluated the extent of impediment to particle transport in CF sputum by the equation 
$\langle\mathrm{MSD}\rangle=4 D_{o} \tau^{\alpha}$, where $D_{o}$ is the $\tau$-independent particle diffusivity and $\alpha$ is the anomalous diffusion exponent that signifies the extent of impediment to particle penetration ( $\alpha=1$ for pure unhindered Brownian diffusion; $\alpha$ approaches zero as obstruction to particle diffusion increases). The average $\alpha$ value was 0.91 for $200 \mathrm{~nm}$ PS-PEG in NAC-treated CF sputum, compared with 0.70 for the same particles in native sputum, suggesting that the transport of $200 \mathrm{~nm}$ PS-PEG is minimally hindered in NAC-treated sputum.

Since fast moving outlier nanoparticles represent a subpopulation that is more likely to readily penetrate the sputum barrier, we characterized the heterogeneity in the transport of individual particles by plotting the distribution of the logarithms of individual particle

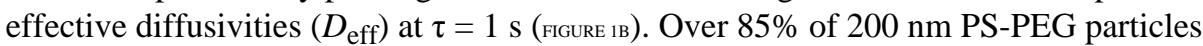
exhibited $D_{\text {eff }}$ greater than $0.1 \mu \mathrm{m}^{2} / \mathrm{s}$ in NAC-treated sputum, a marked increase over the approximately $35 \%$ estimate for the same particles in native sputum. We also classified the mechanisms of particle transport into three non-overlapping transport modes (immobile, hindered and diffusive; where rates of movement, diffusive $>$ hindered $>$ immobile) (FIGURE IC). Based on this analysis, approximately 65 and 35\% of $200 \mathrm{~nm}$ PS-PEG particles were classified as undergoing unhindered diffusion in NAC-treated and native CF sputum, respectively. The average transport rate of the diffusive fraction of $200 \mathrm{~nm}$ PS-PEG particles in NAC-treated sputum is only approximately fourfold slower than that for the same particles in water, which is similar to the average speed previously reported for $200 \mathrm{~nm}$ PSPEG particles in fresh, unaltered CVM obtained from donors with healthy vaginal flora (without CF) [14]. We next estimated the fraction of particles that may penetrate a $10 \mu \mathrm{m}-$ thick CF sputum layer as a function of time, based on fitting the $\tau$-dependent $D_{\text {eff }}$ for individual particles over long time scales to Fick's second law of diffusion (FIGURE2) [13]. Based on this analysis, over $75 \%$ of $200 \mathrm{~nm}$ PS-PEG particles are capable of penetrating across a $10-\mu \mathrm{m}$-thick NAC-treated sputum layer in $20 \mathrm{~min}$, compared with approximately $35 \%$ for the same particles in native sputum ( $\mathrm{p}<0.01)$ (FGURE 2$)$.

The substantial improvement in the transport rates of $200 \mathrm{~nm}$ particles mediated by NAC treatment was evident only with the particles that were densely coated with PEG; the transport of uncoated $200 \mathrm{~nm}$ PS particles was strongly retarded by sputum pretreated with NAC (supplementary movie 4 ). The $\left\langle D_{\text {eff }}\right\rangle$ for uncoated $200 \mathrm{~nm}$ PS particles in NAC-treated sputum $(\tau=1 \mathrm{~s})$ remained approximately 530 -fold slower than the same particles in water (TABle 1). There is little difference in the distribution of individual $D_{\text {eff }}$ for uncoated $200 \mathrm{~nm}$ particles in native and NAC-treated sputum (FGURE 1в), and over $90 \%$ of $200 \mathrm{~nm}$ PS particles in NAC-treated CF sputum were classified as immobilized or strongly hindered (FIGURE IC).

\section{Transport of PEG-coated \& uncoated $500 \mathrm{~nm}$ particles in native \& NAC-treated CF sputum}

We next investigated if adjuvant NAC treatment can also enhance the transport rates of 500 $\mathrm{nm}$ PS-PEG particles in CF sputum. We first measured the transport rates of $500 \mathrm{~nm}$ PS and PS-PEG particles in native sputum. In good agreement with our previous findings, a dense PEG coating only mediated minor improvements in the transport of $500 \mathrm{~nm}$ particles, as reflected by the approximately fivefold greater $\langle$ MSD $\rangle$ at a time scale $(\tau)$ of $1 \mathrm{~s}$ (FIGURE 3A). The average transport rate of the $500 \mathrm{~nm}$ PS-PEG particles remained approximately 350 fold slower than their theoretical rates in water (TABLE 1), indicating that the PEG coating alone on $500 \mathrm{~nm}$ particles was unable to overcome the strong retardation by the sputum mesh.

To enhance the sputum penetration of $500 \mathrm{~nm}$ PS-PEG particles, CF sputum was again pretreated with NAC. The average $\langle\mathrm{MSD}\rangle$ of $500 \mathrm{~nm}$ PS-PEG particles increased threefold upon NAC treatment ( $\tau=1 \mathrm{~s}$ ) (FGGRE $3 \mathrm{~A})$; this improvement was observed in all sputum samples tested $(\mathrm{p}<0.001)$. The $\alpha$ values were 0.46 and 0.73 for PS-PEG particles in native and NAC-treated sputum, respectively (TABLE 1 ), confirming that NAC significantly reduced the diffusional barrier property of CF sputum. Overall, the transport of $500 \mathrm{~nm}$ PS-PEG 
particles in CF sputum pretreated with NAC was slowed by only an average of approximately 100 -fold compared with their theoretical speeds in water.

We observed a substantial fraction of fast moving $500 \mathrm{~nm}$ PS-PEG particles in CF sputum pretreated with NAC; over $20 \%$ of $500 \mathrm{~nm}$ PS-PEG particles exhibited $D_{\text {eff }}(\tau=1 \mathrm{~s})$ larger than $0.1 \mu \mathrm{m}^{2} / \mathrm{s}$ in NAC-treated sputum (FIGURE зв). By contrast, we found no particle displayed $D_{\text {eff }}(\tau=1 \mathrm{~s})$ larger than $0.1 \mu \mathrm{m}^{2} / \mathrm{s}$ in native sputum. Based on transport mode analysis, while over $98 \%$ of $500 \mathrm{~nm}$ PS-PEG particles in native sputum were immobilized or strongly hindered, more than $40 \%$ of $500 \mathrm{~nm}$ PS-PEG particles underwent nearly unhindered diffusion in NAC-treated sputum (FGURe sc), with $\left\langle D_{\text {eff }}\right\rangle$ only approximately sixfold lower than that for the same particles in water (data not shown). The large percentage of $500 \mathrm{~nm}$ PS-PEG undergoing unhindered transport is in good agreement with a substantial fraction of fast moving particles, as shown in the individual $D_{\text {eff }}$ distribution analysis (FIGURE 3B).

Polyethylene glycol coatings were critical for the improved transport of $500 \mathrm{~nm}$ particles mediated by NAC, since uncoated $500 \mathrm{~nm}$ PS particles remained strongly hindered by $\mathrm{CF}$ sputum pretreated with NAC. The difference in $\langle\mathrm{MSD}\rangle$ was not statistically significant for $500 \mathrm{~nm}$ PS particles in native and NAC-treated sputum ( $\tau=1 \mathrm{~s}$ ), owing to the large overlap in the distribution of individual particle $D_{\text {eff }}$ (FIGURE 3А \& зв). Over $95 \%$ of uncoated particles were immobilized or trapped in both native and NAC-treated sputum (FiguRE зC). Overall, the $\left\langle D_{\text {eff }}>\right.$ of uncoated $500 \mathrm{~nm}$ PS particles was approximately 1600- and 1000-fold lower in native and NAC-treated sputum, respectively, compared with the same particles in water (TABLE 1).

Based on our measurements in combination with Fick's second law of diffusion, approximately $30 \%$ of $500 \mathrm{~nm}$ PS-PEG particles were classified as capable of penetrating a $10-\mu \mathrm{m}$-thick NAC-treated sputum layer in $20 \mathrm{~min}$, and approximately $40 \%$ within $2 \mathrm{~h}$ (FIGURE 4). Less than $5 \%$ of $500 \mathrm{~nm}$ PS-PEG particles in native sputum, as well as less than $1 \%$ and approximately $4 \%$ for $500 \mathrm{~nm}$ uncoated particles in native and NAC-treated sputum, respectively, were expected to do so within $20 \mathrm{~min}$. The differences in the estimated penetrable fractions between $500 \mathrm{~nm}$ PS-PEG in NAC-treated sputum and all other conditions were statistically significant $(\mathrm{p}<0.05)$.

\section{D interfiber spacing of native \& NAC-treated CF sputum}

By fitting the measured speeds of 200 and $500 \mathrm{~nm}$ PS-PEG particles, along with published data on transport rate of FITC-dextran, to an obstruction-scaling model, we previously estimated the average distance between mucin fibers (i.e., mesh spacing) of fresh, undiluted and unaltered CF sputum to be approximately $140 \pm 50 \mathrm{~nm}$ [13]. Using the same technique, we found that NAC treatment increased the average mesh spacing of CF sputum samples tested here from $145 \pm 50 \mathrm{~nm}$ to $230 \pm 50 \mathrm{~nm}(\mathrm{p}<0.001)$. The pore sizes were also substantially more heterogeneous in NAC-treated sputum $(50-1300 \mathrm{~nm})$ compared with native sputum $(50-300 \mathrm{~nm})$, with a large fraction of pores in NAC-treated sputum in excess of $300 \mathrm{~nm}$ (FGURE 5A\& $5 B$ ). The increased fraction of larger pores upon NAC treatment was also visually confirmed with scanning electron $\mathrm{m}$ icrographs (FIGURE 5C \& \&D).

\section{Discussion}

Cystic fibrosis sputum represents a tenacious diffusional barrier to aerosol drug and gene carriers due to its elevated viscoelasticity $[11,16,17]$. Previous attempts to overcome the sputum barrier largely focused on using mucolytics to reduce the barrier property of CF sputum, but showed little success [6,7,31-33]. More recently, we have engineered particles with muco-inert coatings, and shown that these nanoparticles can exhibit diffusion in CF sputum at appreciable rates for the first time $[11,13]$. Nevertheless, neither approach alone 
facilitated particle penetration at rates we have previously observed in human CVM obtained from healthy volunteers [14,15], and large fractions of particles remained effectively trapped or greatly slowed in CF sputum. Hindered particle transport is most likely attributed to a mucus mesh that is expected to be substantially denser for CF sputum than CVM. We previously estimated the average mesh spacing of minimally perturbed CF sputum $(\sim 140 \pm 50 \mathrm{~nm})[13]$ to be substantially smaller than that for CVM $(\sim 340 \pm 70 \mathrm{~nm})$ [28]. This difference is likely a consequence of the elevated solid content in CF sputum compared with normal mucus [34].

In the present study, large fractions of nanoparticles are shown to penetrate NAC-treated CF sputum at speeds approaching those in water, most likely enabling much greater fractions of particles to penetrate the thick sputum layer. Improved penetration of nanoparticles through $\mathrm{CF}$ sputum holds important implications for CF therapeutics, including gene therapy. In previous CF gene therapy clinical trials using synthetic nonviral systems, such as liposomeand polymer-based gene carriers, partial correction of the chloride ion imbalance in the nares of CF patients has been observed [35,36]. However, the sputum barrier is not as pronounced in the nares as in the CF airways. In one trial that showed partial restitution of chloride abnormality in the lower $\mathrm{CF}$ airways, there was no evidence of normalization in sodium ion imbalance, which caused CF sputum to remain dehydrated and, hence, muco ciliary clearance impaired [3]. The lack of sodium abnormality restitution likely reflects inadequate gene transfer, since, while complete correction of the chloride ion abnormality can be achieved with as little as 5\% cells expressing functional CFTR, a much larger fraction of cells $(\sim 25 \%)$ must express functional CFTR before sodium abnormality is fully reversed in vitro [37,38]. While poor gene transfer has been attributed by some to limited cellular uptake across the apical membrane, unproductive intracellular trafficking, suboptimal promoters, toxicity and immunological barriers [35,39,40], limited transport of gene carriers across the sputum barrier is increasingly recognized as a critical bottleneck to gene transfer to airway cells $[11,16]$. Enhanced sputum penetration by gene carriers, based on a combined particle surface chemistry and mucolytics strategy, will most likely increase the number of gene carriers that reach target epithelia and, hence, improve CF gene therapy.

The improved particle transport rates observed here are most likely attributed to increased sputum pore sizes, including a substantial fraction of pores $(\sim 12 \%)$ in excess of $500 \mathrm{~nm}$. Larger pores will reduce the steric obstruction that limits particle penetration across $\mathrm{CF}$ sputum. Nevertheless, NAC treatment of sputum only enhanced the penetration rates of particles that do not adhere to sputum, indicating that coating particles with a muco-inert surface is essential for rapid particle penetration across sputum, even when the sputum mesh spacing is greatly increased. Our observations suggest that previous failures to observe marked improvements in particle transport upon treatment with mucolytics may be attributed to extensive particle adhesion to the residual sputum mesh. For example, Shute and coworkers demonstrated that NAC did not improve the transport of PS particles in synthetic CF sputum fluids (i.e., physical mixture of porcine mucins, DNA and actin filaments) [41]; PS particles are strongly mucoadhesive $[13,14]$. It is also worth noting that previous studies investigating the effects of another common mucolytic, rhDNase, on particle transport in CF sputum were performed with mucoadhesive PS particles, which may explain the negligible benefits observed [6,7].

It was not obvious, a priori, that NAC would improve the diffusion of nanoparticles in CF sputum since it is controversial whether mucin is even the primary constituent of the sputum mesh. Previous work has suggested CF sputum to be a mesh network composed primarily of DNA and actin, with almost no intact mucins or little mucin content [42]. By contrast, others have reported that mucin is the most abundant macromolecule in CF sputum, and that the higher mucin content in CF causes an increase in the bulk rheological properties of CF 
sputum $[7,16]$. Another study demonstrated that the weight-averaged molecular weight of mucins in CF sputum was found to be similar to that in normal mucus [43,44], which suggests the mucins in CF sputum may not be substantially fragmented. Whether mucin comprises the sputum mesh is perhaps dependent on the patient and the disease stage [45]. In the CF sputum samples we used, we found the mucin content to be substantially greater than the DNA content (data not shown), suggesting that mucins were the primary structural component of the sputum mesh in this study.

It remains unclear exactly how NAC increases the pore sizes in sputum. Dawson and coworkers previously showed that rhDNase offered little to no improvement in nanoparticle transport in CF sputum [6]. In fact, elimination of fast and slow moving outlier particles upon treatment of sputum with rhDNase led the authors to suggest that rhDNase may induce a higher microviscosity (viscosity in the watery channels) in CF sputum due to the release of soluble DNA fragments. By contrast, NAC did not increase the microviscosity of CF sputum, since the diffusive fraction of $200 \mathrm{~nm}$ PS-PEG particles in NAC-treated sputum exhibited speeds only approximately fourfold slower than the same particles in pure water, which is comparable to a previous observation that FITC-dextran is slowed approximately threefold in native CF sputum compared with in water [5]. That NAC did not increase the sputum microviscosity was unexpected a priori, since NAC cleaves disulfide crosslinks and, therefore, was expected to release mucins into the low viscosity interstitial fluids between sputum mesh; soluble mucin molecules that diffused into the pores of the sputum mesh would be expected to increase the microviscosity of CF sputum far more than the relatively small DNA fragments liberated by rhDNase (longer polymers increase viscosity to a much greater extent than shorter polymers at the same concentration [46]). One possible explanation that can reconcile our various findings is that NAC may not induce release of soluble mucin. Instead, cleaved mucin fibers may remain associated with the sputum mesh via entanglements, hydro phobic interactions or other adhesive interactions [47], which led to larger pores without releasing soluble mucins that can increase the viscosity of interstitial fluid.

In the present study, a final NAC concentration of $20 \mathrm{mM}$ in sputum was chosen based on previous work by King and coworkers that estimated the maximum concentration of NAC to be approximately $20 \mathrm{mM}$ in dog airways (assuming a 10\% deposition in the trachea and major bronchi via a metered dose inhaler) [48]. This NAC dose decreased mucus viscoelasticity and improved clearance without any evident side effects. A higher dose of mucolytics may be delivered to the airways; the deposition of nacystelyn, a lysine derivative of NAC, was approximately three-times greater when administered to healthy volunteers via a dry powder inhaler [49]. The NAC dose used in this study did not destroy the CF sputum network structure, as shown by scanning electron micrographs and as suggested by the fact that uncoated particles were effectively immobilized by adhesion to the visco elastic residual sputum mesh. A tenfold lower NAC dose $(2 \mathrm{mM})$ also enhanced particle penetration across $\mathrm{CF}$ sputum to a similar extent; however, further reduction in NAC dose $(0.2 \mathrm{mM})$ substantially reduced the adjuvant effects of NAC (data not shown).

Rapid sputum penetration of substantial fractions of $500 \mathrm{~nm}$ particles may have important implications for numerous CF therapeutic applications, since larger particles offer improved encapsulation of a wider array of drugs, as well as controlled drug-release kinetics over longer times [50,51]. It is worth noting that PEG coating on nanoparticles most likely resists adsorption of NAC, and thus the presence of NAC should not affect the degradation kinetics of the biodegradable nanoparticles or the rate of drug release. Use of other mucolytic agents, which act on other major sputum constituents, such as DNA and filamentous actins, in combination with NAC, may further enhance the penetration of large nanoparticles in CF sputum. Hypertonic saline, known to augment airway surface liquid volume and decrease 
airway surface liquid viscosity [52], should also be considered as an adjuvant to improve the sputum penetration of nanoparticles in CF patients.

\section{Conclusion}

$\mathrm{N}$-acetyl cysteine treatment of CF sputum greatly increased the mesh spacing, thereby allowing large fractions of polymeric nanoparticles to rapidly penetrate NAC-treated CF sputum if the nanoparticles possess non-mucoadhesive surfaces. Non-mucoadhesive nanoparticles were achieved here through coatings with a dense brush layer of lowmolecular-weight PEG. This combined mucolytics/nanoparticle-coating strategy has significant therapeutic implications for $\mathrm{CF}$ and possibly other pulmonary diseases characterized by excess mucus production, since the sputum barrier has been increasingly recognized as a major hurdle to airway gene and drug delivery.

\section{Future perspective}

A combined mucolytics/nanoparticle-coating strategy that enables particles as large as 500 $\mathrm{nm}$ to penetrate sputum layers is introduced here. We expect our approach will facilitate improved retention and distribution of drug carriers in the CF lung, enabling sustained delivery of a wide range of therapeutics. This approach may also facilitate more efficient gene therapy of the CF lungs.

\section{Executive summary}

- Existing approaches to improve particle diffusion across cystic fibrosis (CF) sputum, either by using mucolytic adjuvants or reducing particle adhesion to the sputum mesh, do not facilitate rapid diffusion of particles at rates comparable to those previously observed for nanoparticles in human cervicovaginal mucus.

- $\quad$ Simultaneously reducing the $\mathrm{CF}$ sputum barrier with $N$-acetyl cysteine (NAC) and reducing particle-mucin adhesion with muco-inert coatings synergistically enhances particle penetration across CF sputum.

- Polyethylene glycol-coated $200 \mathrm{~nm}$ particles can rapidly penetrate NACtreated sputum with average speeds approaching their theoretical speeds in water.

- Combining polyethylene glycol coatings and adjuvant NAC strategy allows sputum penetration by larger particles (i.e., $500 \mathrm{~nm}$ ) that are otherwise trapped in CF sputum.

- NAC most likely enhances the diffusion of nanoparticles in sputum by increasing the mesh spacings of the dense CF sputum mesh.

- Rapid diffusion is critically dependent on the presence of a muco-inert coating on nanoparticles; NAC alone only marginally improved the sputum penetration of conventional nanoparticles.

\section{Supplementary Material}

Refer to Web version on PubMed Central for supplementary material. 


\section{Acknowledgments}

The authors would like to thank Meghan Ramsay and Sharon Watts at the Johns Hopkins Adult Cystic Fibrosis Center for cystic fibrosis sputum collection.

Financial \& competing interests disclosure

This work was supported by the NIH (NIH 1R01 EB003558 and P01 HL51811), the Cystic Fibrosis Foundation (CFF HANES08G0) and a postdoctoral fellowship from the Croucher Foundation to Samuel K Lai. The content is solely the responsibility of the authors and does not necessarily represent the official views of the NIH.

The mucus penetrating particle technology described in this publication is being developed by Kala Pharmaceuticals. Hanes is cofounder of and serves on the Board of Directors of Kala. Hanes owns company stock, which is subject to certain restrictions under University policy. The terms of this arrangement are being managed by the Johns Hopkins University in accordance with its conflict of interest policies. The authors have no other relevant affiliations or financial involvement with any organization or entity with a financial interest in or financial conflict with the subject matter or materials discussed in the manuscript apart from those disclosed.

No writing assistance was utilized in the production of this manuscript.

\section{Bibliography}

Papers of special note have been highlighted as:

- of interest

m of considerable interest

1. Davis PB, Drumm M, Konstan MW. Cystic fibrosis. Am. J. Respir. Crit. Care Med. 1996; 154(5): 1229-1256. [PubMed: 8912731]

2. Rubin BK. Mucus structure and properties in cystic fibrosis. Paediatr. Respir. Rev. 2007; 8(1):4-7. [PubMed: 17419972]

3. Boucher RC. Evidence for airway surface dehydration as the initiating event in CF airway disease. J. Intern. Med. 2007; 261(1):5-16. [PubMed: 17222164]

4. Kitson C, Angel B, Judd D, et al. The extra- and intracellular barriers to lipid and adenovirusmediated pulmonary gene transfer in native sheep airway epithelium. Gene Ther. 1999; 6(4):534546. [PubMed: 10476213]

5. Braeckmans K, Peeters L, Sanders NN, De Smedt SC, Demeester J. Three-dimensional fluorescence recovery after photobleaching with the confocal scanning laser microscope. Biophys. J. 2003; 85(4): 2240-2252. [PubMed: 14507689]

6. Dawson M, Wirtz D, Hanes J. Enhanced viscoelasticity of human cystic fibrotic sputum correlates with increasing microheterogeneity in particle transport. J. Biol. Chem. 2003; 278(50):5039350401. [PubMed: 13679362]

7m. Sanders NN, De Smedt SC, Van Rompaey E, Simoens P, De Baets F, Demeester J. Cystic fibrosis sputum: a barrier to the transport of nanospheres. Am. J. Respir. Crit. Care Med. 2000; 162(5):1905-1911. [PubMed: 11069833] [An elegant report highlighting the diffusional barrier property of cystic fibrosis (CF) sputum]

8. Laube BL. The expanding role of aerosols in systemic drug delivery, gene therapy, and vaccination. Respir. Care. 2005; 50(9):1161-1176. [PubMed: 16122400]

9. Garcia-Contreras L, Hickey AJ. Aerosol treatment of cystic fibrosis. Crit. Rev. Ther. Drug Carrier Syst. 2003; 20(5):317-356. [PubMed: 14959788]

10. Montier T, Delepine P, Pichon C, Ferec C, Porteous DJ, Midoux P. Non-viral vectors in cystic fibrosis gene therapy: progress and challenges. Trends Biotechnol. 2004; 22(11):586-592. [PubMed: 15491803]

11. Lai SK, Wang YY, Hanes J. Mucus-penetrating nanoparticles for drug and gene delivery to mucosal tissues. Adv. Drug Deliv. Rev. 2009; 61(2):158-171. [PubMed: 19133304] [A recent review on developing synthetic nanoparticles for transmucosal drug/gene delivery] 
12m. Tang BC, Dawson M, Lai SK, et al. Biodegradable polymer nanoparticles that rapidly penetrate the human mucus barrier. Proc. Natl Acad. Sci. USA. 2009; 106(46):19268-19273. [PubMed: 19901335] [Demonstrates minimally mucoadhesive biodegradable drug carriers exhibit effective diffusivities approximately 100 -fold faster than conventional mucoadhesive nanoparticles]

13m. Suk JS, Lai Sk, Wang YY, et al. The penetration of fresh undiluted sputum expectorated by cystic fibrosis patients by non-adhesive polymer nanoparticles. Biomaterials. 2009; 30(13):25912597. [PubMed: 19176245] [Demonstrates that nanoparticles engineered with minimally mucoadhesive surfaces can exhibit enhanced diffusion in purulent CF sputum]

14m. Lai SK, O'Hanlon DE, Harrold S, et al. Rapid transport of large polymeric nanoparticles in fresh undiluted human mucus. Proc. Natl Acad. Sci. USA. 2007; 104(5):1482-1487. [PubMed: 17244708] [Demonstrates that nanoparticles can be engineered to rapidly penetrate fresh human mucus]

15匹. Wang YY, Lai SK, Suk JS, Pace A, Cone R, Hanes J. Addressing the PEG mucoadhesivity paradox to engineer nanoparticles that 'slip' through the human mucus barrier. Angew. Chem. Int. Ed. 2008; 47(50):9726-9729. [An important study that describes polyethylene glycol (PEG) coating chemistry that effectively reduces mucoadhesion of nanoparticles compared with prior reports of PEG as a mucoadhesive polymer]

16m. Sanders N, Rudolph C, Braeckmans K, De Smedt SC, Demeester J. Extracellular barriers in respiratory gene therapy. Adv. Drug Deliv. Rev. 2009; 61(2):115-127. [PubMed: 19146894] [A comprehensive review of extracellular barriers to CF gene therapy]

17. Tobacman JK. Does deficiency of arylsulfatase b have a role in cystic fibrosis? Chest. 2003; 123(6):2130-2139. [PubMed: 12796199]

18. Henke MO, Ratjen F. Mucolytics in cystic fibrosis. Paediatr. Respir. Rev. 2007; 8(1):24-29. [PubMed: 17419975]

19. Sanders NN, Van Rompaey E, De Smedt SC, Demeester J. On the transport of lipoplexes through cystic fibrosis sputum. Pharm. Res. 2002; 19(4):451-456. [PubMed: 12033379]

20. Sheffner AL, Medler EM, Jacobs LW, Sarett HP. The in vitro reduction in viscosity of human tracheobronchial secretions by acetylcysteine. Am. Rev. Respir. Dis. 1964; 90:721-729. [PubMed: 14211458]

21. Gallon AM. Evaluation of nebulised acetylcysteine and normal saline in the treatment of sputum retention following thoracotomy. Thorax. 1996; 51(4):429-432. [PubMed: 8733499]

22. App EM, Baran D, Dab I, et al. Dose-finding and 24-h monitoring for efficacy and safety of aerosolized nacystelyn in cystic fibrosis. Eur. Respir. J. 2002; 19(2):294-302. [PubMed: 11866009]

23. Suh J, Dawson M, Hanes J. Real-time multiple-particle tracking: applications to drug and gene delivery. Adv. Drug Deliv. Rev. 2005; 57(10):1551-1551.

24. Suk JS, Suh J, Lai SK, Hanes J. Quantifying the intracellular transport of viral and nonviral gene vectors in primary neurons. Exp. Biol. Med. (Maywood). 2007; 232(3):461-469. [PubMed: 17327481]

25. Suh J, Wirtz D, Hanes J. Efficient active transport of gene nanocarriers to the cell nucleus. Proc. Natl Acad. Sci. USA. 2003; 100(7):3878-3882. [PubMed: 12644705]

26. Apgar J, Tseng Y, Fedorov E, Herwig MB, Almo SC, Wirtz D. Multiple-particle tracking measurements of heterogeneities in solutions of actin filaments and actin bundles. Biophys. J. 2000; 79(2):1095-1106. [PubMed: 10920039]

27. Suh J, Choy KL, Lai SK, et al. PEGylation of nanoparticles improves their cytoplasmic transport. Int. J. Nanomedicine. 2007; 2(4):735-741. [PubMed: 18203439]

28m. Lai SK, Wang YY, Hida K, Cone R, Hanes J. Nanoparticles reveal that human cervicovaginal mucus is riddled with pores larger than viruses. Proc. Natl Acad. Sci. USA. 2010; 107(2):598603. [PubMed: 20018745] [The microstructure in human cervicovaginal mucus based on nonperturbing nanoparticle probes that reveals the pores are markedly larger than those in $\mathrm{CF}$ sputum, motivating the use of mycolytics to increase the pore sizes in CF sputum]

29. Olmsted SS, Padgett JL, Yudin AI, Whaley KJ, Moench TR, Cone RA. Diffusion of macromolecules and virus-like particles in human cervical mucus. Biophys. J. 2001; 81(4):19301937. [PubMed: 11566767] 
30. Shen H, Hu YY, Saltzman WM. DNA diffusion in mucus: effect of size, topology of DNAs, and transfection reagents. Biophys. J. 2006; 91(2):639-644. [PubMed: 16632500]

31. Ferrari S, Kitson C, Farley R, et al. Mucus altering agents as adjuncts for nonviral gene transfer to airway epithelium. Gene Ther. 2001; 8(18):1380-1386. [PubMed: 11571577]

32m. Kushwah R, Oliver JR, Cao H, Hu J. Nacystelyn enhances adenoviral vector-mediated gene delivery to mouse airways. Gene Ther. 2007; 14(16):1243-1248. [PubMed: 17525704] [Demonstrates that mucolytics can improve airway gene transfer in vivo]

33. Stern M, Caplen NJ, Browning JE, et al. The effect of mucolytic agents on gene transfer across a CF sputum barrier in vitro. Gene Ther. 1998; 5(1):91-98. [PubMed: 9536269]

34. Matsui H, Verghese MW, Kesimer M, et al. Reduced three-dimensional motility in dehydrated airway mucus prevents neutrophil capture and killing bacteria on airway epithelial surfaces. J. Immunol. 2005; 175(2):1090-1099. [PubMed: 16002710]

35m. Griesenbach U, Alton EW. Gene transfer to the lung: lessons learned from more than 2 decades of CF gene therapy. Adv. Drug Deliv. Rev. 2009; 61(2):128-139. [PubMed: 19138713] [A recent comprehensive review of clinical trials for CF gene therapy]

36. Pringle IA, Hyde SC, Gill DR. Non-viral vectors in cystic fibrosis gene therapy: recent developments and future prospects. Expert Opin. Biol. Ther. 2009; 9(8):991-1003. [PubMed: 19545217]

37. Johnson LG, Boyles SE, Wilson J, Boucher RC. Normalization of raised sodium absorption and raised calcium-mediated chloride secretion by adenovirus-mediated expression of cystic fibrosis transmembrane conductance regulator in primary human cystic fibrosis airway epithelial cells. J. Clin. Invest. 1995; 95(3):1377-1382. [PubMed: 7533790]

38. Zhang L, Button B, Gabriel SE, et al. CFTR delivery to $25 \%$ of surface epithelial cells restores normal rates of mucus transport to human cystic fibrosis airway epithelium. PLoS Biol. 2009; 7(7):E1000155. [PubMed: 19621064]

39. Ziady AG, Davis PB. Current prospects for gene therapy of cystic fibrosis. Curr. Opin. Pharmacol. 2006; 6(5):515-521. [PubMed: 16890018]

40. Schultz BR, Chamberlain JS. Recombinant adeno-associated virus transduction and integration. Mol. Ther. 2008; 16(7):1189-1199. [PubMed: 18500252]

41. Brougton-Head VJ, Smith JR, Shur J, Shute JK. Actin limits enhancement of nanoparticle diffusion through cystic fibrosis sputum by mucolytics. Pulm. Pharmacol. Ther. 2007; 20(6):708717. [PubMed: 17055310]

42. Voynow JA, Rubin BK. Mucins, mucus, and sputum. Chest. 2009; 135(2):505-512. [PubMed: 19201713]

43. Thornton DJ, Davies JR, Kraayenbrink M, Richardson PS, Sheehan JK, Carlstedt I. Mucus glycoproteins from 'normal' human tracheobronchial secretion. Biochem. J. 1990; 265(1):179_ 186. [PubMed: 2302164]

44. Thornton DJ, Sheehan JK, Lindgren H, Carlstedt I. Mucus glycoproteins from cystic fibrotic sputum. Macromolecular properties and structural 'architecture'. Biochem. J. 1991; 276(Pt 3): 667-675. [PubMed: 2064605]

45. Henke MO, John G, Germann M, Lindemann H, Rubin BK. MUC5AC and MUC5B mucins increase in cystic fibrosis airway secretions during pulmonary exacerbation. Am. J. Respir. Crit. Care Med. 2007; 175(8):816-821. [PubMed: 17255563]

46. Shizuo H, Yo T, Toshiharu T. Rheology of concentrated polymer solution: dependence of viscosity on concentration and molecular weight. J. Soc. Mater. Sci. (Japan). 1967; 16(166):520-522.

47. Lai SK, Wang YY, Cone R, Wirtz D, Hanes J. Altering mucus rheology to 'solidify' human mucus at the nanoscale. PLoS ONE. 2009; 4(1):E4294. [PubMed: 19173002]

48. Tomkiewicz RP, App EM, De Sanctis GT, et al. A comparison of a new mucolytic Nacetylcysteine l-lysinate with $\mathrm{N}$-acetylcysteine: airway epithelial function and mucus changes in dog. Pulm. Pharmacol. 1995; 8(6):259-265. [PubMed: 8819180]

49. Vanderbist F, Wery B, Baran D, Van Gansbeke B, Schoutens A, Moes AJ. Deposition of nacystelyn from a dry powder inhaler in healthy volunteers and cystic fibrosis patients. Drug Dev. Ind. Pharm. 2001; 27(3):205-212. [PubMed: 11291200]

50. Langer R. Drug delivery and targeting. Nature. 1998; 392(6679 Suppl.):5-10. [PubMed: 9579855] 
51. Yoo HS, Oh JE, Lee KH, Park TG. Biodegradable nanoparticles containing doxorubicin-PLGA conjugate for sustained release. Pharm. Res. 1999; 16(7):1114-1118. [PubMed: 10450940]

52. Levin MH, Sullivan S, Nielson D, Yang B, Finkbeiner WE, Verkman AS. Hypertonic saline therapy in cystic fibrosis: evidence against the proposed mechanism involving aquaporins. J. Biol. Chem. 2006; 281(35):25803-25812. [PubMed: 16829520] 


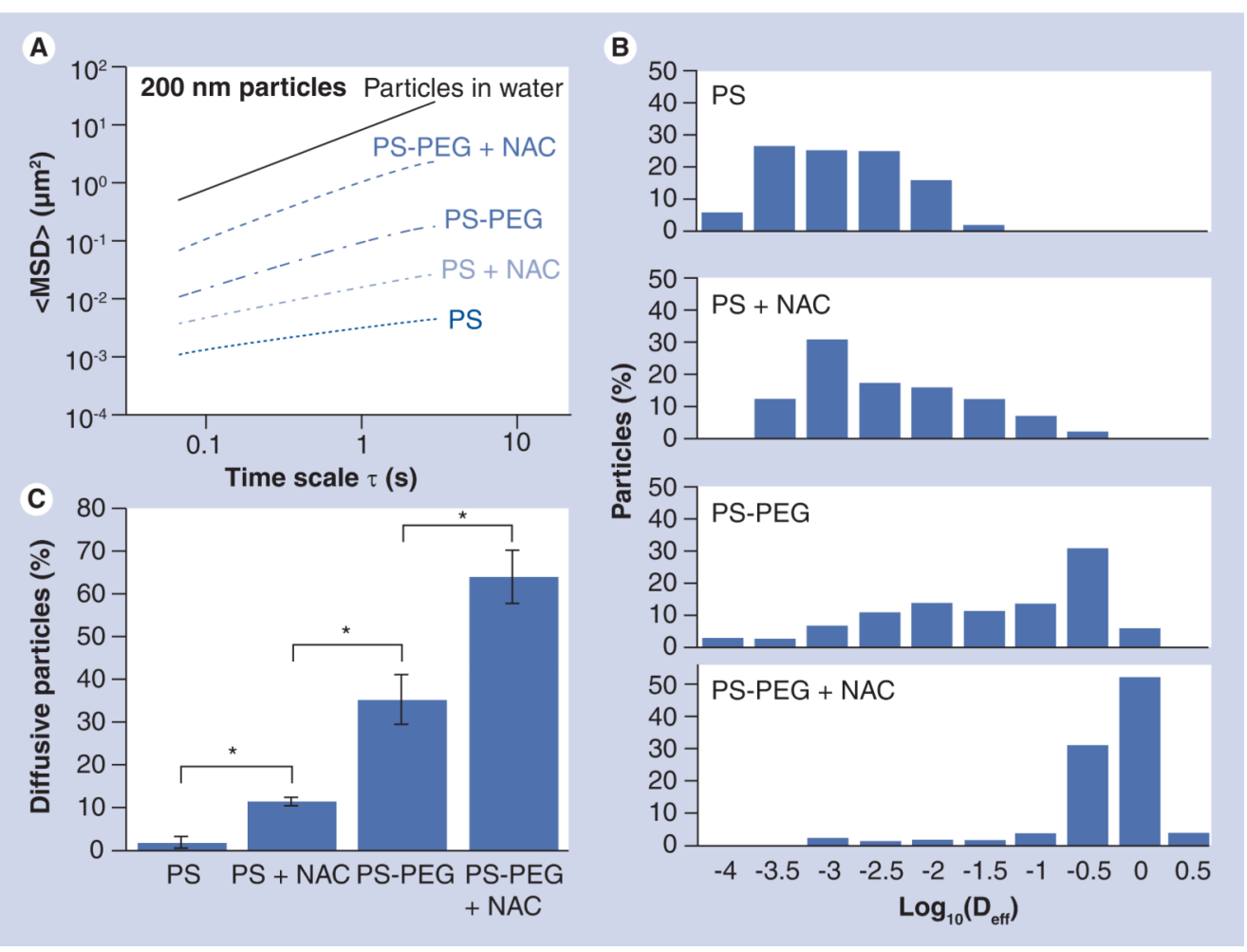

Figure 1. Transport of $200 \mathrm{~nm}$ uncoated (PS) and polyethylene glycol-coated (PS-PEG) polystyrene nanoparticles in native or $N$-acetyl cysteine-treated cystic fibrosis sputum (A) $\langle$ MSD $>$ as a function of time scale $(\tau)$. (B) Distribution of the logarithms of individual particle $\mathrm{D}_{\text {eff }}$ at a time scale of $1 \mathrm{~s}$. (C) Percentage of particles that undergo unhindered diffusive transport in native or NAC-treated cystic fibrosis sputum. Data represents three individual experiments, with $n>200$ particles for each experiment.

*Statistically significant differences $(\mathrm{p}<0.05)$.

$\mathrm{D}_{\text {eff }}$ : Effective diffusivity; MSD: Mean square displacement; NAC: $N$-acetyl cysteine; PEG: Polyethylene glycol; PS: Polystyrene. 


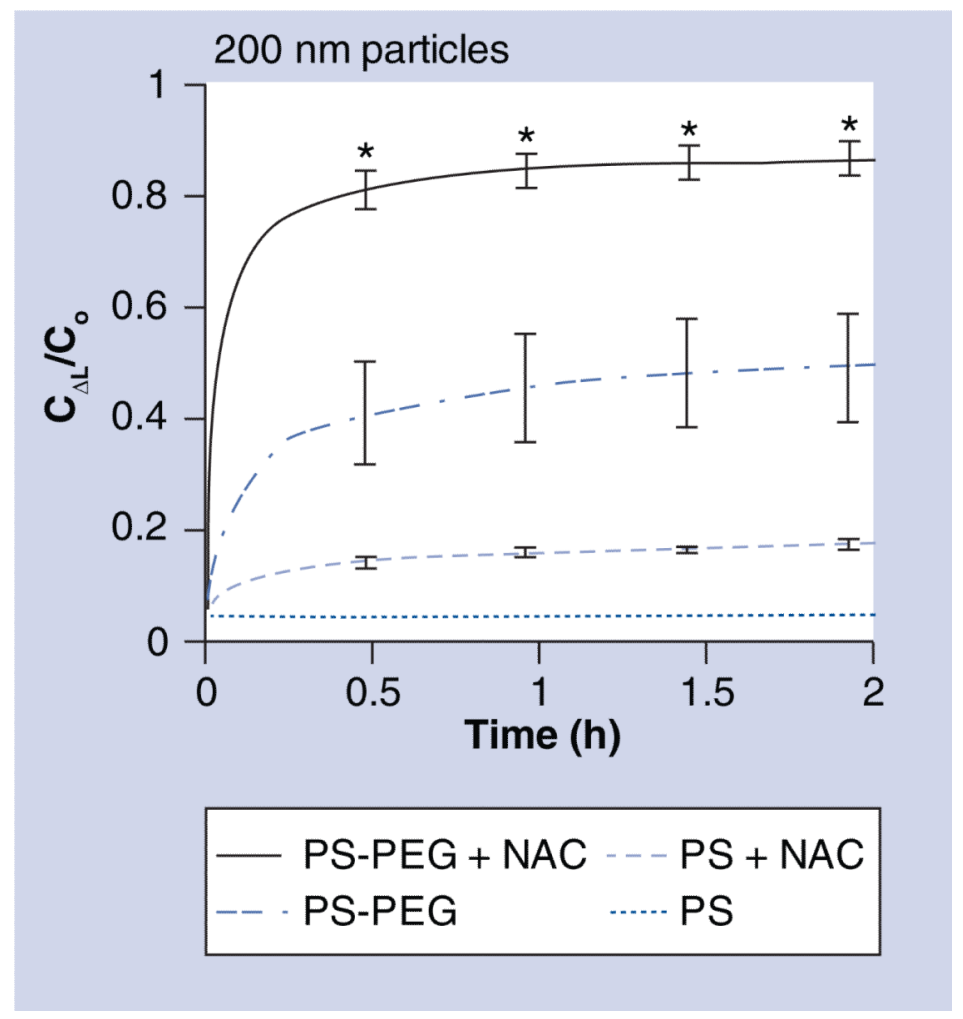

Figure 2. Fraction of $200 \mathrm{~nm}$ uncoated (PS) and polyethylene glycol-coated (PS-PEG) particles that are expected to penetrate a $\mathbf{1 0} \boldsymbol{\mu m}$ cystic fibrosis sputum layer over time

Ratio of particle concentration at $\Delta \mathrm{L}=10 \mu \mathrm{m}$ deep $\left(\mathrm{C}_{\Delta \mathrm{L}}\right)$ to initial particle concentration at the surface $\left(C_{0}\right)$ over time. Data represents three individual experiments, with $n>200$ particles for each experiment. Error bars indicate standard error of the mean.

*Statistically significant differences compared with three other conditions $(\mathrm{p}<0.01)$. C: Concentration; NAC: $N$-acetyl cysteine; PEG: Polyethylene glycol; PS: Polystyrene. 


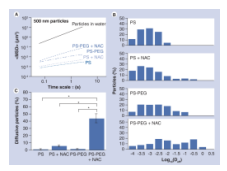

Figure 3. Transport of $500 \mathrm{~nm}$ uncoated (PS) and polyethylene-coated (PS-PEG) polystyrene nanoparticles in native or $\mathrm{N}$-acetyl cysteine-treated cystic fibrosis sputum

(A) $\langle$ MSD $>$ as a function of time scale $(\tau)$. (B) Distribution of the logarithms of individual particle $\mathrm{D}_{\text {eff }}$ at a time scale of $1 \mathrm{~s}$. (C) Percentage of particles that undergo unhindered diffusive transport in native or NAC-treated CF sputum. Data represents three individual experiments, with $\mathrm{n}>200$ particles for each experiment.

*Statistically significant differences $(\mathrm{p}<0.05)$.

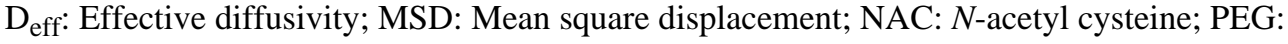
Polyethylene glycol; PS: Polystyrene. 


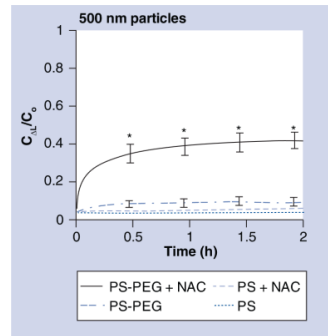

Figure 4. Fraction of $500 \mathrm{~nm}$ uncoated (PS) and polyethylene glycol-coated (PS-PEG) particles that are expected to penetrate $\mathbf{1 0} \boldsymbol{\mu m}$ cystic fibrosis sputum layer over time Ratio of particle concentration at $\Delta \mathrm{L}=10 \mu \mathrm{m}$ deep $\left(\mathrm{C}_{\Delta \mathrm{L}}\right)$ to initial particle concentration at the surface $\left(C_{0}\right)$ over time. Data represents three individual experiments, with $n>200$ particles for each experiment. Error bars indicate standard error of the mean.

*Statistically significant differences compared with three other conditions $(\mathrm{p}<0.01)$. C: Concentration; NAC: $N$-acetyl cysteine; PEG: Polyethylene glycol; PS: Polystyrene. 

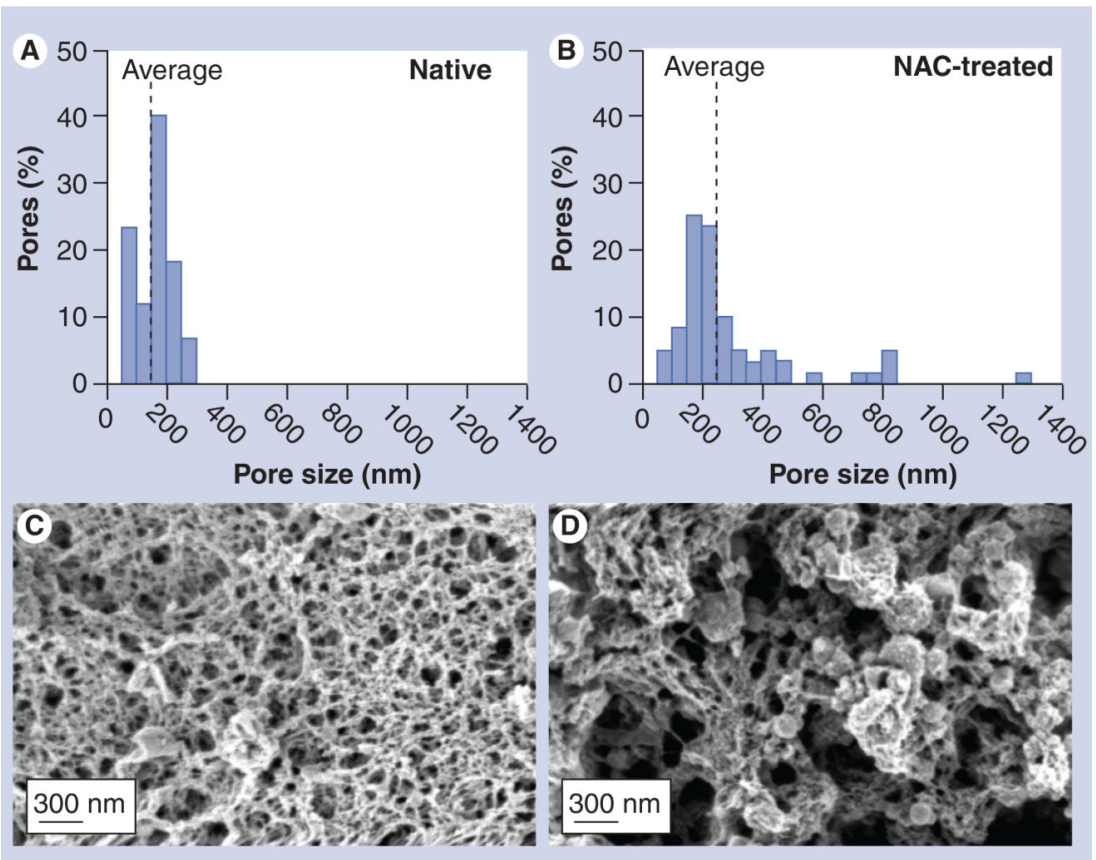

Figure 5. 3D microstructures of cystic fibrosis sputum

(A \& B) Pore size distribution of (A) native and (B) NAC-treated cystic fibrosis sputum. The average pore sizes predicted for 200 and $500 \mathrm{~nm}$ polystyrene-polyethylene glycol nanoparticles by the obstruction-scaling model are $145 \pm 50 \mathrm{~nm}$ and $230 \pm 50 \mathrm{~nm}$ for native and NAC-treated CF sputum, respectively. (C \& D) Scanning electron micrographs of (C) native and (D) NAC-treated CF sputum. There is an increase in the fraction of larger pores upon NAC treatment.

NAC: $N$-acetyl cysteine. 


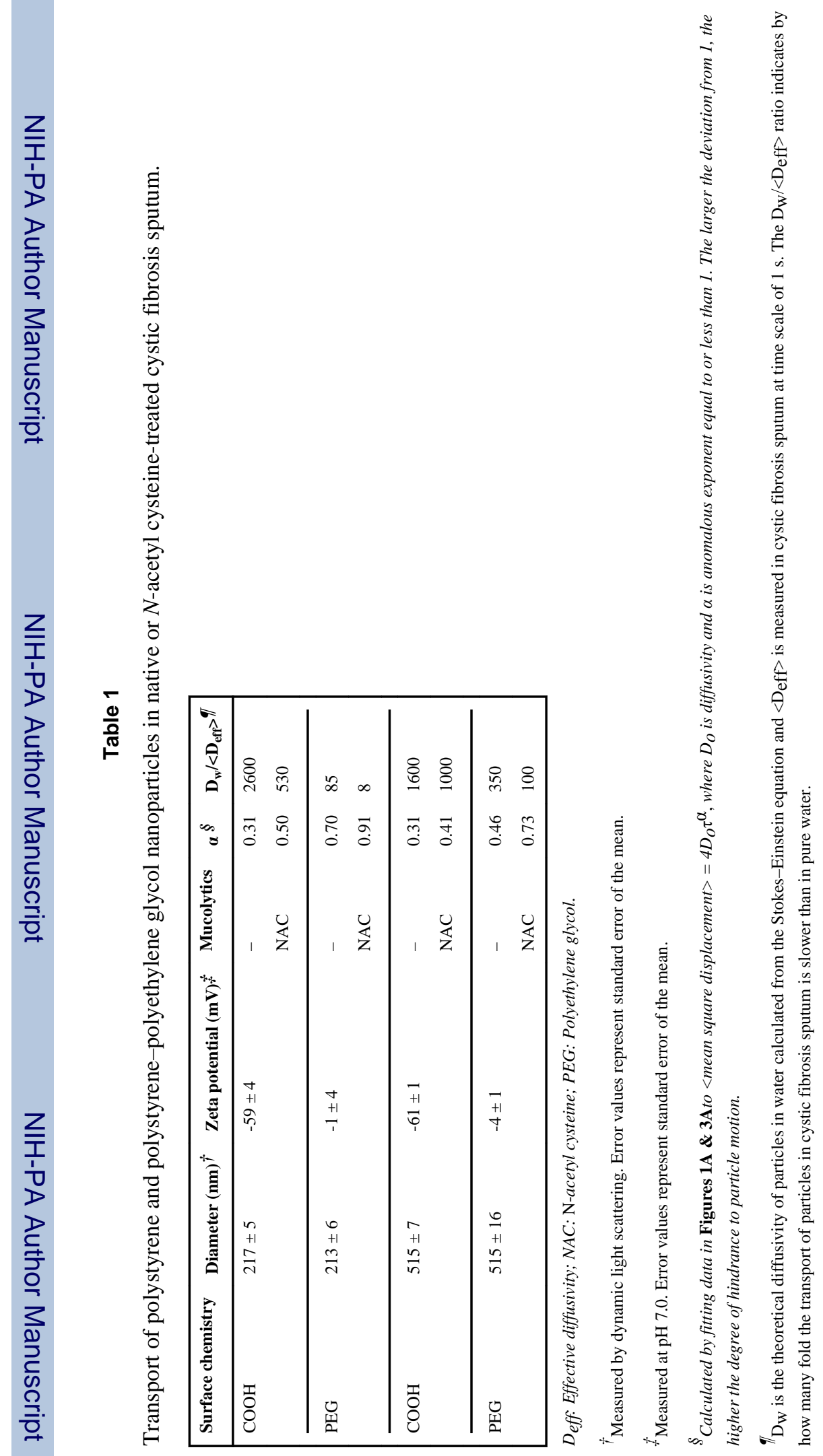

Nanomedicine (Lond). Author manuscript; available in PMC 2011 December 1. 